\title{
Influence of Geometric Parameters On Mechanical Response of Concentric Cylinders With Centrosymmetric Lattice
}

Dejun Jia ( $\nabla$ junge_tt@163.com )

Dalian Maritime University

Fanchun Li

Dalian Maritime University

Zhijie Liu

Dalian Maritime University

Yuan Zhang

Dalian Maritime University

\section{Research Article}

Keywords: Centrosymmetric Lattice, Concentric Cylinders, Lightweight design, Axisymmetric load

Posted Date: September 22nd, 2021

DOI: https://doi.org/10.21203/rs.3.rs-895628/v1

License: (9) This work is licensed under a Creative Commons Attribution 4.0 International License. Read Full License 


\section{Influence of Geometric Parameters on Mechanical Response of}

\section{Concentric Cylinders with Centrosymmetric Lattice}

Dejun Jia ${ }^{1}$, Fanchun Li, Zhijie Liu, Yuan Zhang

(School of Ship and Ocean Engineering, Dalian Maritime University, Dalian, 116026)

Abstract: A design method of centrosymmetric lattice structure is proposed. The centrosymmetric lattice is applied to the lightweight design of concentric cylindrical structures. Combining homogenization method and finite element method, the advantage of centrosymmetric lattice concentric cylinder in reducing the maximum stress is verified, and the stress distribution of centrosymmetric lattice concentric cylinder is more uniform along the circumference; two kinds of centrosymmetric lattice solids are selected, and the parametric method is used to study the influence of three parameters on the static response of a centrosymmetric lattice concentric cylinder under axisymmetric surface pressure. The results show that the maximum stress of the central symmetric concentric cylinder is less than that of the parallel array lattice cylinder under axisymmetric surface pressure; the stress distribution of the cylinder under axisymmetric load is periodic along the circumference, and the stress distribution of the concentric cylinder is more uniform than that of the parallel array lattice while the centrosymmetric lattice is applied to the lightweight design of concentric cylinder; the maximum stress increases with the increase of inside thickness and decreases with the increase of outside thickness; the increase of lattice section size will reduce the stress of outside and lattice of concentric cylinder, while the maximum stress of inside is less affected by the size of lattice section.

Keywords: Centrosymmetric Lattice; Concentric Cylinders; Lightweight design; Axisymmetric load.

\section{Introduction}

With the increasing demand for lightweight design of components in many industries and the continuous development of metal additive manufacturing technology, in recent years, lightweight structures including metal lattice structures have been widely used in aerospace, medical devices and other mechanical engineering related fields ${ }^{[1]-[15]}$. The application of lattice structure in lightweight design of parts can not only reduce the structural quality of components, but also adjust the geometric size and distribution of lattice structure in parts according to the specific design requirements, so as to change the equivalent elastic modulus and structural stiffness of components, so as to change the mechanical response of parts. In particular, in the field of aerospace, combined with additive manufacturing technology, the application of lattice in the lightweight design of aircraft structure and parts can greatly reduce the structural mass of aircraft and increase the effective carrying of aircraft ${ }^{[16]-[18]}$.

In order to make the lattice structure have better mechanical properties, scholars have carried out a lot of research on lattice structure performance analysis, optimization design and manufacturing technology ${ }^{[19]-[21]}$. The above research work studies the lattice structure from different aspects, and provides technical reference for the optimization design of lattice structure. However, the lattice structures mentioned in these studies are usually obtained by parallel arrays rather than structure adaptive. [22]-[25] and other studies applied the self-adaptive lattice in the

1 Corresponding to Dejun Jia: junge_tt@163.com 
lightweight design of the structure. In addition, compared with the parallel array lattice, the structural adaptive lattice has better structural integrity in irregular lightweight area, which can effectively reduce the structural imperfection and further eliminate the stress concentration.

Compared with the irregular structure, such as the compressor impeller, the revolution structure has regular geometric shape. However, if the parallel array points are used in the lightweight design of this kind of revolution structure, when the axisymmetric load is applied, the stress and deformation of the structure will be uneven along the circumference. In this case, an obvious asymmetric deformation will occur in the structure, which makes the stress distribution more uneven $^{[16]-[18]}$.

Centrosymmetric lattice can be a kind of adaptive lattice for revolution structure. Centrosymmetric lattice is a kind of lattice structure, which is obtained by arranging a single lattice element around a fixed axis at an equal angle. When a revolution structure, such as a compressor impeller, is subjected to an axisymmetric load or a load with the characteristic of uniform distribution along the circumference of the revolution structure, if the centrosymmetric lattice is used instead of the parallel array lattice for lightweight design, its stress distribution and deformation distribution are relatively uniform.

Since centrosymmetric lattice is a special form of structural adaptive lattice, some design methods for structural adaptive lattice can be used in lightweight design of revolution structure. However, because there is no specific consideration of the particularity of centrosymmetric lattice structure compared with the general structure adaptive lattice structure, the lightweight design method of centrosymmetric lattice structure considering the particularity of revolution structure may have better performance. In addition, there are few researches on the parametric design of adaptive lattice structure, especially on the revolving structures. However, this kind of research can evaluate the influence of lattice structure parameters on the mechanical response of lightweight structure, and provide technical reference for structural optimization design.

Based on the current research status, this paper proposes a design method of centrosymmetric lattice for revolution structure, and evaluates the advantages and disadvantages of centrosymmetric lattice relative to parallel array lattice in lightweight design of revolution structure based on homogenization method. Then, two kinds of centrosymmetric lattice structures are selected and applied to the lightweight design of concentric cylinders. Based on parametric design and finite element method, the influence of lightweight area percentage and lattice size on stress response of concentric cylinder after lightweight design is discussed, which provides technical reference for lightweight design of revolution structure.

2. Mechanical response analysis of parallel array and centrosymmetric lattice based on homogenization method

As shown in Figure 1, the centrosymmetric lattice has been applied to the lightweight design of revolution structures ${ }^{[17]}$. Zhang redesigned a simple cubic lattice structure to make it a self-adaptive lattice of revolution structure, and applied it to the lightweight design of concentric cylinder and compressor impeller. When the centrosymmetric lattice is applied to lightweight design of concentric cylinder, the maximum radial stress and circumferential stress can be effectively reduced by $6 \%-20 \%$. 


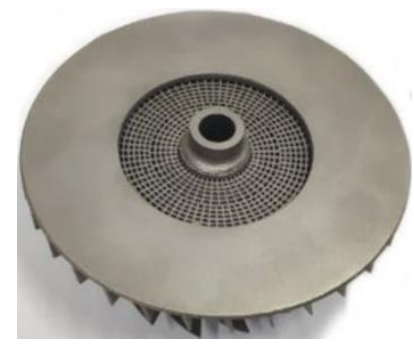

Figure 1 Lattice compressor impeller

In addition to the lattice used by Zhang, there are 11 kinds of lattice structures commonly used in structural lightweight design as shown in Figure 2. These lattices have excellent mechanical properties, which can make the structure have the designed structural stiffness and equivalent modulus while realizing the lightweight design of the structure ${ }^{[26]}$.

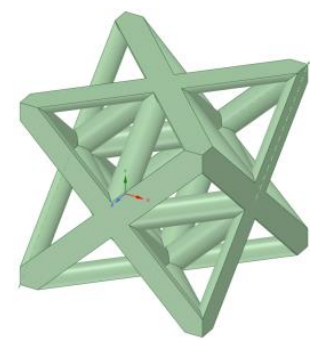

(a) No.1

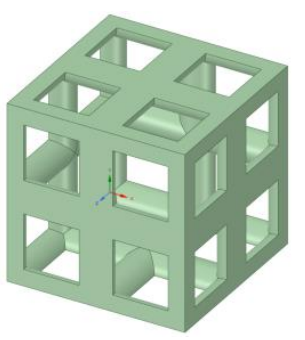

(e) No.5

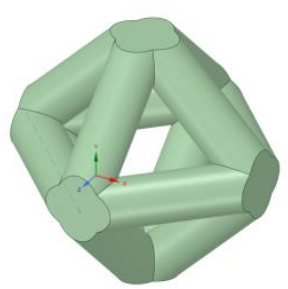

(i) No.9

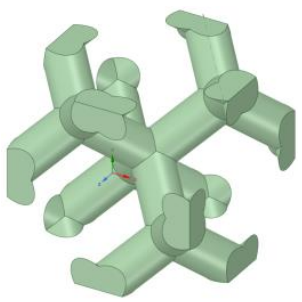

(b) No.2

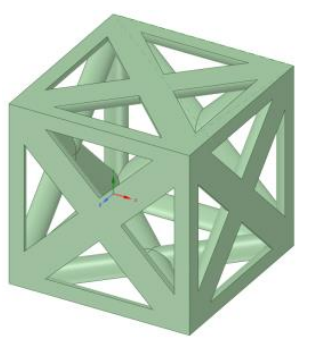

(f) No.6

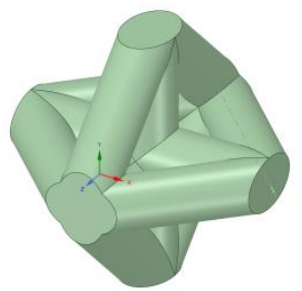

(j) No.10

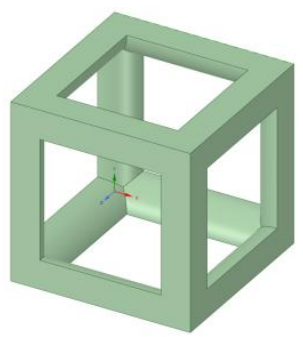

(c) No.3

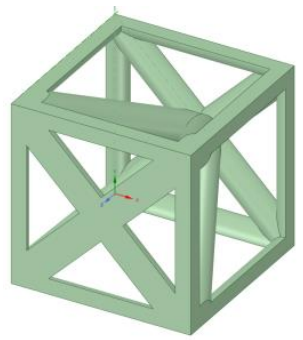

(g) No.7

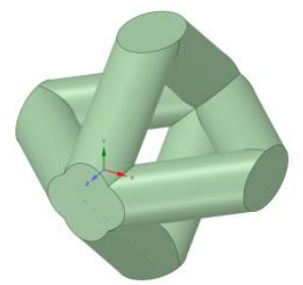

(k) No.11

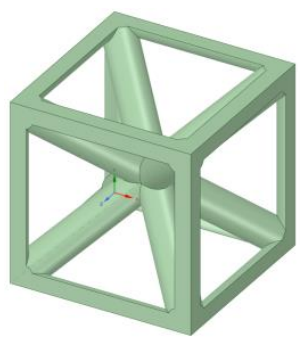

(d) No.4

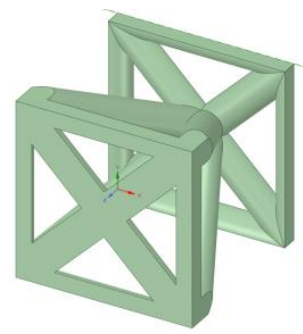

(h) No.8

Figure 2 Eleven kinds of lattice

In order to study the advantages and disadvantages of centrosymmetric lattice structure compared with parallel array lattice structure in lightweight design of revolution structure, two different types of lattice need to be applied to the same typical body of revolution in the lightweight design of structure (such as concentric cylinder). The mechanical response of the revolving body structure with two kinds of lattice for lightweight design is analyzed under the action of uniformly distributed load along the circumference. Because the single column of lattice structure is smaller than the revolution structure, if the lattice structure is directly applied to the lightweight design of revolution structure according to the actual geometric appearance, and 
modeling and finite element analysis are carried out, the number of nodes in the finite element model will be higher in the calculation process, and there will be more unnecessary nodes in the non lightweight area. In this case, the finite element calculation process takes too long. Yang et $\mathrm{al}^{[27]-[28]}$. introduced the homogenization method to equivalent the lattice structure. Under the premise of ensuring the required calculation accuracy, the mechanical response of lightweight lattice structure can be evaluated more quickly. In this section, a similar method is used to homogenize the lattice structure to obtain the equivalent elastic modulus of the lattice structure. Then, the homogenized material is applied to the lightweight design of concentric cylinder combined with finite element method, and the mechanical response of concentric cylinder under axisymmetric load is analyzed.

Based on the homogenization method of Yang ${ }^{[27]-[28]}$, the orthotropic linear elastic characteristics of a single lattice can be obtained approximately.

This section considers loads in six directions, namely, loads in three tensile directions (x, y, z) and loads in three shear directions (XY, YZ, XZ), as shown in Figure 3. The corresponding macroscopic strain is applied in each direction, and the reaction force of the representation volume element (RVE) interface is used to obtain the stiffness matrix, and then the required engineering constants can be obtained.

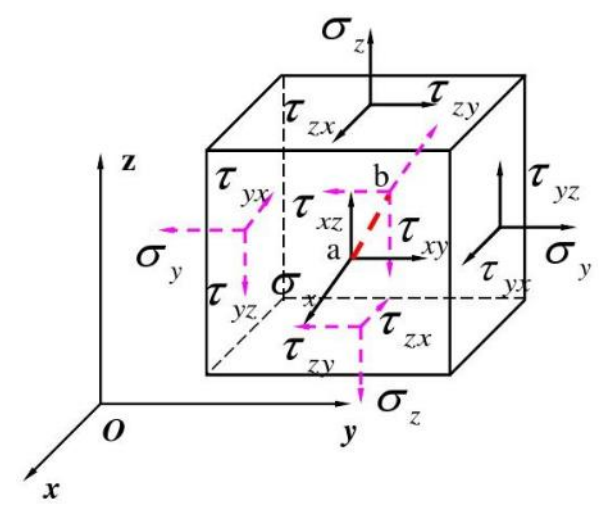

Figure 3 Directions in an element

Firstly, the mechanical equation under tensile load in $x$ direction is considered. For orthotropic materials, the following relationship exists:

$$
\left[\begin{array}{c}
\sigma_{x} \\
\sigma_{y} \\
\sigma_{z} \\
\sigma_{x y} \\
\sigma_{y z} \\
\sigma_{x z}
\end{array}\right]=\left[\begin{array}{ccccc}
D_{11} & D_{12} & D_{13} & \\
D_{21} & D_{22} & D_{23} & & \\
D_{31} & D_{32} & D_{33} & \\
& & \multicolumn{2}{c}{D_{44}} & \\
& & \multicolumn{2}{c}{D_{55}} \\
& & & & \\
& & & & D_{66}
\end{array}\right]\left[\begin{array}{l}
\varepsilon_{x} \\
\varepsilon_{y} \\
\varepsilon_{z} \\
\gamma_{x y} \\
\gamma_{y z} \\
\gamma_{x z}
\end{array}\right]
$$

where $\sigma_{x}, \sigma_{y}, \sigma_{z}, \sigma_{x y}, \sigma_{y z}$ and $\sigma_{x z}$ are the stress in each direction, $D_{i j}$ are the coefficient in the stiffness matrix $(i, j=1,2,3,4,5,6), \varepsilon_{x}, \varepsilon_{y}, \varepsilon_{z}, \gamma_{x y}, \gamma_{y z}, \gamma_{x z}$ are the strain in each direction. If the strain in the $\mathrm{X}$ direction is fixed to $\varepsilon_{x}=0.001$ and all other strains are set 
to 0 , the first column of the stiffness matrix can be obtained

$$
\left[\begin{array}{l}
D_{11} \\
D_{21} \\
D_{31} \\
0 \\
0 \\
0
\end{array}\right]=\frac{1}{0.001}\left[\begin{array}{l}
\sigma_{x} \\
\sigma_{y} \\
\sigma_{z} \\
\sigma_{x y} \\
\sigma_{y z} \\
\sigma_{x z}
\end{array}\right]
$$

Considering that the lattice structure is usually periodic in geometry, the volume of RVE is assumed to be $\left[0, L_{x}\right] \times\left[0, L_{y}\right] \times\left[0, L_{z}\right]$. Here, ${ }^{L_{x}}, L_{y}$ and $L_{z}$ are the lengths of rve in $x, y$ and $z$ directions. In the plane normal to the $x$-axis, enforce

$$
\begin{aligned}
& u_{x}\left(L_{x}, y, z\right)=u_{x}(0, y, z)+\varepsilon L_{x} \\
& u_{y}\left(L_{x}, y, z\right)=u_{y}(0, y, z) \\
& u_{z}\left(L_{x}, y, z\right)=u_{z}(0, y, z)
\end{aligned}
$$

In the plane normal to the $y$-axis, enforce

$$
\begin{aligned}
& u_{x}\left(x, L_{y}, z\right)=u_{x}(x, 0, z) \\
& u_{y}\left(x, L_{y}, z\right)=u_{y}(x, 0, z) \\
& u_{z}\left(x, L_{y}, z\right)=u_{z}(x, 0, z)
\end{aligned}
$$

In the plane normal to the $z$-axis, enforce

$$
\begin{aligned}
& u_{x}\left(x, y, L_{z}\right)=u_{x}(x, y, 0) \\
& u_{y}\left(x, y, L_{z}\right)=u_{y}(x, y, 0) \\
& u_{z}\left(x, y, L_{z}\right)=u_{z}(x, y, 0)
\end{aligned}
$$

In addition to these periodic conditions, it is necessary to restrict the rigid body motion in the calculation process. Here, enforce

$$
\begin{aligned}
& u_{x}(\text { a point with } x=0)=0 \\
& u_{y}(\text { a point with } y=0)=0 \\
& u_{z}(\text { a point with } z=0)=0
\end{aligned}
$$

There are alternatives to these periodic boundary conditions. Unless there exist enough symmetries, these alternatives lead to boundary effects. On periodic structures, periodic boundary conditions should be used.

To compute macroscopic stresses, the forces on the top faces are integrated. Consider $\sigma_{x}$. The force in the $x$-direction at the face $x=L_{x}$ is integrated. $\sigma_{x}$ is obtained by normalizing with the face area. $\sigma_{y}$ and $\sigma_{z}$ are obtained similarly. The entries for $D_{11}, D_{21}$ and $D_{31}$ in the stiffness matrix are easily obtained. By repeating the steps for all the other load cases, all the entries for the 
stiffness matrix are obtained. The stiffness matrix is inverted to obtain the compliance matrix

$$
[C]=[D]^{-1}
$$

Finally, the engineering constants $E_{x}, E_{y}, E_{z}, G_{x y}, G_{y z}, G_{x z}, v_{x y}, v_{y z}$ and $v_{x z}$ are computed from the relationship

$$
[C]=\left[\begin{array}{ccccc}
\frac{1}{E_{x}} \frac{-v_{y x}}{E_{y}} & \frac{-v_{z x}}{E_{z}} & & \\
\frac{-v_{x y}}{E_{x}} \frac{1}{E_{y}} & \frac{-v_{z y}}{E_{z}} & & \\
\frac{-v_{x z}}{E_{x}} \frac{-v_{y z}}{E_{y}} & \frac{1}{E_{z}} & & \\
& & & \frac{1}{G_{x y}} & \\
& & & \frac{1}{G_{y z}} \\
& & & \frac{1}{G_{x z}}
\end{array}\right]
$$

The corresponding material modulus of the homogenized lattice is shown in Table 1. The lattice here is made of titanium alloy.

Table 1 equivalent elastic modulus $(\mathrm{Pa})$ and Poisson's ratio of the material corresponding to the homogenized lattice

\begin{tabular}{|c|c|c|c|c|c|}
\hline$E_{x}$ & $3.6409 \times 10^{9}$ & $G_{x y}$ & $2.39 \times 10^{9}$ & $v_{x y}$ & 0.33092 \\
\hline$E_{y}$ & $3.6409 \times 10^{9}$ & $G_{y z}$ & $2.39 \times 10^{9}$ & $v_{y z}$ & 0.33093 \\
\hline$E_{z}$ & $3.6409 \times 10^{9}$ & $G_{x z}$ & $2.39 \times 10^{9}$ & $v_{x z}$ & 0.33093 \\
\hline \multicolumn{6}{|c|}{ Lattice No. 2} \\
\hline$E_{x}$ & $2.2599 \times 10^{9}$ & $G_{x y}$ & $2.1154 \times 10^{9}$ & $v_{x y}$ & 0.39791 \\
\hline$E_{y}$ & $2.2592 \times 10^{9}$ & $G_{y z}$ & $2.1154 \times 10^{9}$ & $v_{y z}$ & 0.39877 \\
\hline$E_{z}$ & $2.2584 \times 10^{9}$ & $G_{x z}$ & $2.1154 \times 10^{9}$ & $v_{x z}$ & 0.39863 \\
\hline \multicolumn{6}{|c|}{ Lattice No.3 } \\
\hline$E_{x}$ & $8.7993 \times 10^{9}$ & $G_{x y}$ & $5.2281 \times 10^{8}$ & $v_{x y}$ & 0.099347 \\
\hline$E_{y}$ & $8.7991 \times 10^{9}$ & $G_{y z}$ & $5.2281 \times 10^{8}$ & $v_{y z}$ & 0.099586 \\
\hline
\end{tabular}

Lattice No.1 


\begin{tabular}{|c|c|c|c|c|c|}
\hline$E_{z}$ & $8.7989 \times 10^{9}$ & $G_{x z}$ & $5.2281 \times 10^{8}$ & $v_{x z}$ & 0.099584 \\
\hline \multicolumn{6}{|c|}{ Lattice No.4 } \\
\hline$E_{x}$ & $4.1222 \times 10^{9}$ & $G_{x y}$ & $2.2151 \times 10^{9}$ & $v_{x y}$ & 0.30893 \\
\hline$E_{y}$ & $4.1223 \times 10^{9}$ & $G_{y z}$ & $2.2151 \times 10^{9}$ & $v_{y z}$ & 0.30884 \\
\hline$E_{z}$ & $4.1224 \times 10^{9}$ & $G_{x z}$ & $2.2151 \times 10^{9}$ & $v_{x z}$ & 0.30885 \\
\hline \multicolumn{6}{|c|}{ Lattice No.5 } \\
\hline$E_{x}$ & $8.9329 \times 10^{9}$ & $G_{x y}$ & $5.7912 \times 10^{8}$ & $v_{x y}$ & 0.10215 \\
\hline$E_{y}$ & $8.9329 \times 10^{9}$ & $G_{y z}$ & $5.7912 \times 10^{8}$ & $v_{y z}$ & 0.1022 \\
\hline$E_{z}$ & $8.9328 \times 10^{9}$ & $G_{x z}$ & $5.7912 \times 10^{8}$ & $v_{x z}$ & 0.1022 \\
\hline \multicolumn{6}{|c|}{ Lattice No.6 } \\
\hline$E_{x}$ & $5.8763 \times 10^{9}$ & $G_{x y}$ & $1.7556 \times 10^{9}$ & $v_{x y}$ & 0.2346 \\
\hline$E_{y}$ & $5.8763 \times 10^{9}$ & $G_{y z}$ & $1.7556 \times 10^{9}$ & $v_{y z}$ & 0.23461 \\
\hline$E_{z}$ & $5.8763 \times 10^{9}$ & $G_{x z}$ & $1.7556 \times 10^{9}$ & $v_{x z}$ & 0.23461 \\
\hline \multicolumn{6}{|c|}{ Lattice No.7 } \\
\hline$E_{x}$ & $4.2452 \times 10^{9}$ & $G_{x y}$ & $2.2307 \times 10^{9}$ & $v_{x y}$ & 0.40526 \\
\hline$E_{y}$ & $4.2452 \times 10^{9}$ & $G_{y z}$ & $1.4089 \times 10^{9}$ & $v_{y z}$ & 0.094748 \\
\hline$E_{z}$ & $3.7472 \times 10^{9}$ & $G_{x z}$ & $1.4089 \times 10^{9}$ & $v_{x z}$ & 0.094749 \\
\hline \multicolumn{6}{|c|}{ Lattice No.8 } \\
\hline$E_{x}$ & $4.7235 \times 10^{9}$ & $G_{x y}$ & $2.4573 \times 10^{9}$ & $v_{x y}$ & 0.39203 \\
\hline$E_{y}$ & $4.7236 \times 10^{9}$ & $G_{y z}$ & $1.489 \times 10^{9}$ & $v_{y z}$ & 0.23569 \\
\hline$E_{z}$ & $1.3276 \times 10^{9}$ & $G_{x z}$ & $1.489 \times 10^{9}$ & $v_{x z}$ & 0.23569 \\
\hline \multicolumn{6}{|c|}{ Lattice No.9 } \\
\hline$E_{x}$ & $4.3837 \times 10^{9}$ & $G_{x y}$ & $2.2955 \times 10^{9}$ & $v_{x y}$ & 0.30023 \\
\hline$E_{y}$ & $4.3837 \times 10^{9}$ & $G_{y z}$ & $2.2955 \times 10^{9}$ & $v_{y z}$ & 0.30022 \\
\hline$E_{z}$ & $4.3837 \times 10^{9}$ & $G_{x z}$ & $2.2955 \times 10^{9}$ & $v_{x z}$ & 0.30023 \\
\hline
\end{tabular}




\begin{tabular}{cccccc}
\hline \multicolumn{6}{c}{ Lattice No.10 } \\
\hline$E_{x}$ & $6.1154 \times 10^{9}$ & $G_{x y}$ & $1.747 \times 10^{8}$ & $v_{x y}$ & -0.058042 \\
$E_{y}$ & $6.1155 \times 10^{9}$ & $G_{y z}$ & $2.5092 \times 10^{9}$ & $v_{y z}$ & 0.33888 \\
$E_{z}$ & $5.9664 \times 10^{9}$ & $G_{x z}$ & $2.5092 \times 10^{9}$ & $v_{x z}$ & 0.33889 \\
\hline & & \multicolumn{2}{c}{ Lattice No.11 } & & \\
\hline$E_{x}$ & $2.7312 \times 10^{9}$ & $G_{x y}$ & $1.7232 \times 10^{8}$ & $v_{x y}$ & -0.38018 \\
$E_{y}$ & $2.7313 \times 10^{9}$ & $G_{y z}$ & $3.078 \times 10^{9}$ & $v_{y z}$ & 0.5487 \\
$E_{z}$ & $3.6422 \times 10^{9}$ & $G_{x z}$ & $3.078 \times 10^{9}$ & $v_{x z}$ & 0.54873 \\
\hline
\end{tabular}

Because the homogenized material has the properties of orthotropic composites, therefore, if the lattice structure is applied to the lightweight design of concentric cylinder in the way of centrosymmetric array, the equivalent elastic modulus of the material has the same value along the circumference. The concentric cylinder with parallel array lattice for lightweight design has different elastic modulus along the circumference. The distribution of elastic modulus of concentric cylinder with centrosymmetric lattice is equal along the circumference. On the circumference of the same radius, the value of elastic modulus in the radial direction is the value of $E_{x}$. For the concentric cylinder with parallel array, the radial elastic modulus of the material is different at the same radius, the variation of the value with the angle is shown in Figure 4. Here, Figure (a) shows the change of material radial elastic modulus with angle at the same radius of the concentric cylinder with parallel array lattice which is lightweight designed by using lattice No.1, and Figure (b) shows the change of material radial elastic modulus with angle at the same radius of the concentric cylinder with parallel array lattice which is lightweight designed by using lattice No.3,

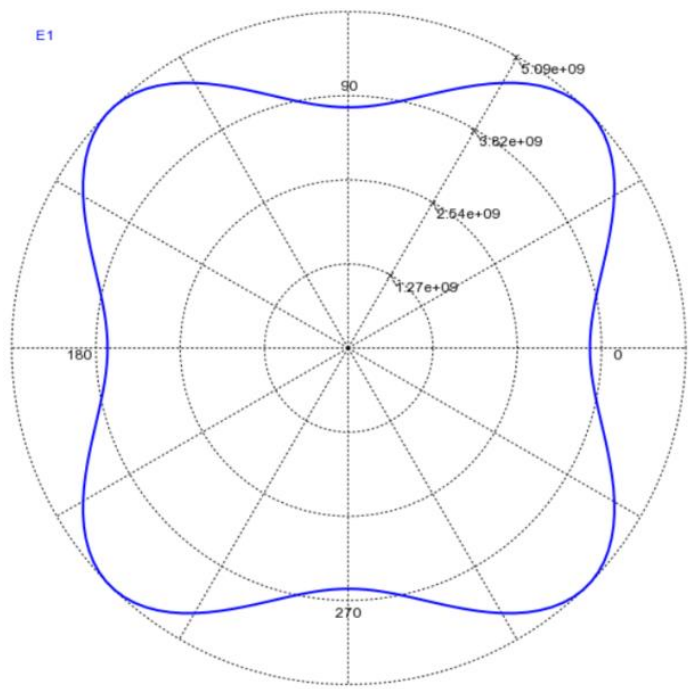

(a)

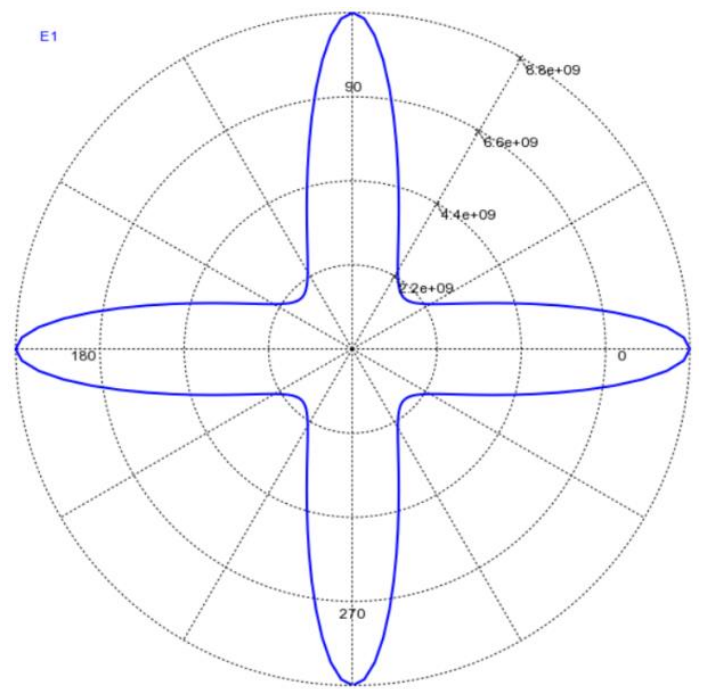

(b)

Figure 4 Change of material properties of concentric cylinder with parallel array lattice with angle 
After defining the material properties of the concentric cylinder, in order to define the constraint conditions in the finite element analysis, a quarter cylinder is selected as the analysis object in this section. The circumferential displacement of the two sections of the concentric cylinder is zero, and a radial surface pressure is applied to the outer cylinder surface. Figure 5 shows the maximum stress and displacement of a concentric cylinder under $1000 \mathrm{~Pa}$ surface pressure. The maximum values of stress and displacement of concentric cylinder after homogenization of centrosymmetric lattice structure, and the maximum values of stress and displacement of concentric cylinder after homogenization of parallel array lattice structure are shown respectively. It can be found from the data in Figure 5 that the maximum structural stress of concentric cylinder under radial surface pressure is less than that of concentric cylinder with parallel array lattice in lightweight design. Among them, in the maximum stress of concentric cylinder designed with No.3, No.5, No.10 and No.11 lattice, the structural stress of uniform cylinder with parallel array lattice is significantly higher than that of uniform cylinder with centrosymmetric lattice.

On the other hand, it can be seen from Figure 5 (b) that the relationship between the maximum displacement and the maximum stress of two kinds of concentric cylinders designed with centrosymmetric lattice and parallel array lattice for lightweight design is slightly different: In some cases (No.1, No.2, No.4 lattice is used for lightweight design), the maximum displacement of concentric cylinder structure with centrosymmetric lattice is higher than that of concentric cylinder structure with parallel array lattice; in some cases (No.6, No.7, No.8, No.9 lattice is used for lightweight design), the maximum displacement of concentric cylinder structure with centrosymmetric lattice is slightly close to that of concentric cylinder structure with parallel array lattice. In particular, when No.3, No.5, No.10 and No.11 lattice are applied to the lightweight design of concentric cylinder, the maximum displacement of concentric cylinder with parallel array lattice is significantly higher than that of concentric cylinder with centrosymmetric lattice.

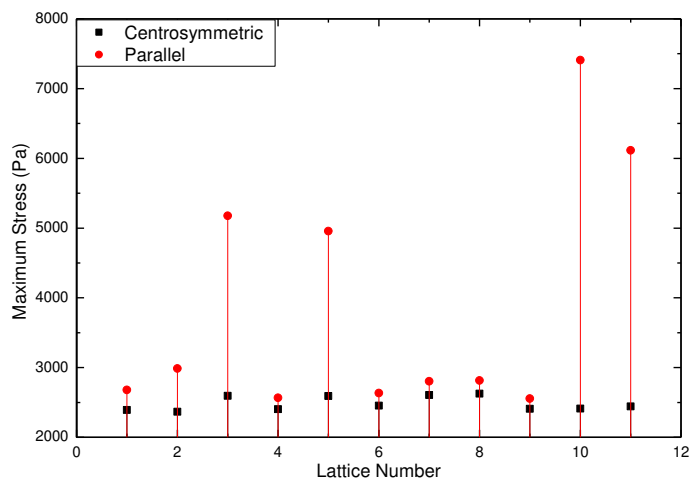

(a)

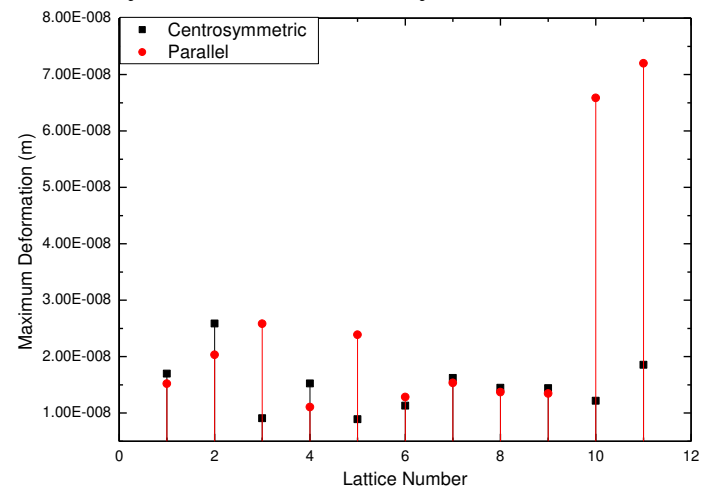

(b)

Figure 5 Maximum stress and maximum displacement

Figure 6 and 7 shows the structural stress and displacement nephogram of concentric cylinder with centrosymmetric lattice and concentric cylinder with parallel array lattice, which are respectively designed and homogenized by using the 11 kinds of lattice in Figure 2. It can be seen from Figure 6 that the stress distribution of the homogenized concentric cylinder with centrosymmetric lattice is uniform along the circumferential direction and increases along the direction pointing to the center of the circle. In addition, it can be seen from the displacement distribution that the displacement distribution of the concentric cylinder with parallel array lattice 
for lightweight design is not uniform along the circumferential direction under the action of uniformly distributed load. In this case, the concentric cylinder will produce a non axisymmetric deformation. This phenomenon will further aggravate the uneven distribution of concentric cylinder materials. If the motion state of concentric cylinder or revolving body with other structural forms is fixed axis rotation, the structure will produce an eccentric load due to its unbalanced mass in the process of fixed axis rotation, which will aggravate the vibration of the structure and further increase the unbalanced load of the structure. Therefore, compared with the parallel array lattice, the stress distribution and mass distribution of the structure are more conducive to the structural safety when the centrosymmetric lattice is applied to the structural lightweight design.

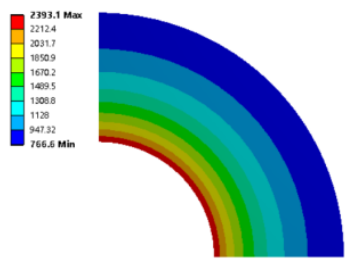

(a) Lattice 1 centrosymmetric

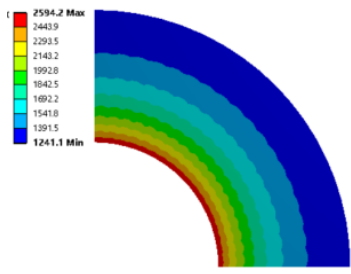

(e) Lattice 3

centrosymmetric

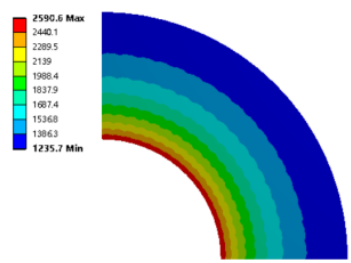

(i) Lattice 5

centrosymmetric

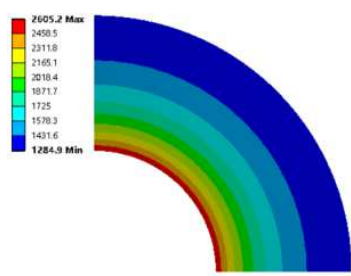

(m) Lattice 7 centrosymmetric

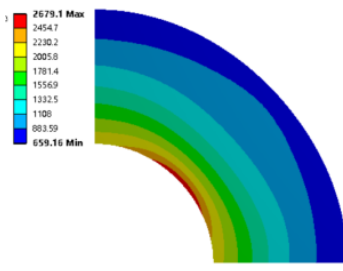

(b) Lattice 1 parallel

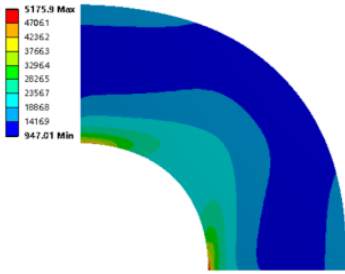

(f) Lattice 3 parallel

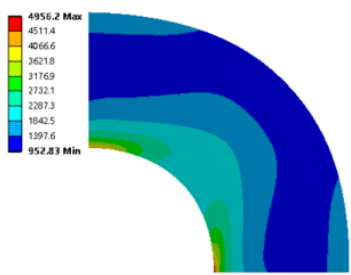

(j) Lattice 5 parallel

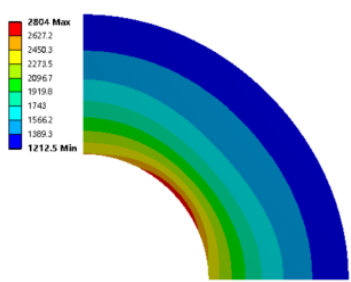

(n) Lattice 7 parallel

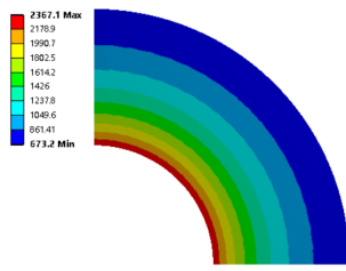

(c) Lattice 2 centrosymmetric

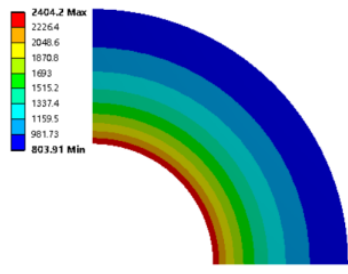

(g) Lattice 4 centrosymmetric

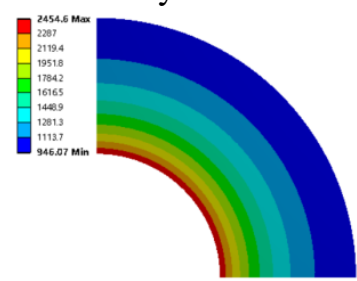

(k) Lattice 6 centrosymmetric

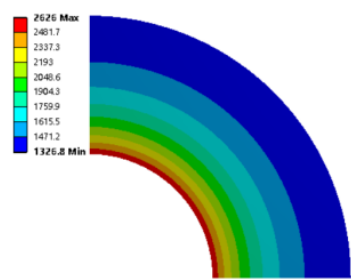

(o) Lattice 8 centrosymmetric

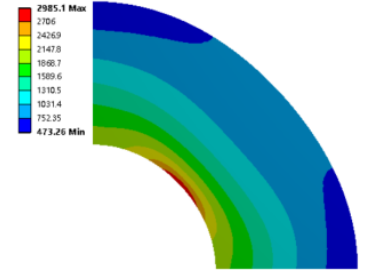

(d) Lattice 2 parallel

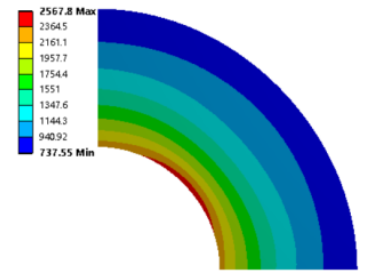

(h) Lattice 4 parallel

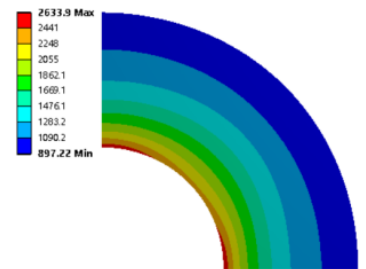

(1) Lattice 6 parallel

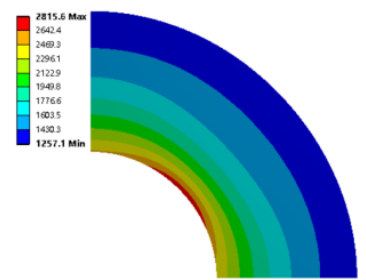

(p) Lattice 8 parallel 


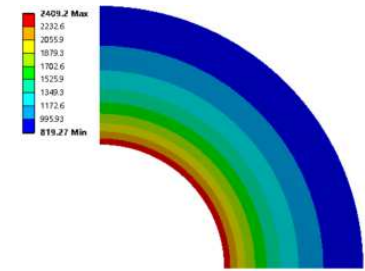

(q) Lattice 9

centrosymmetric

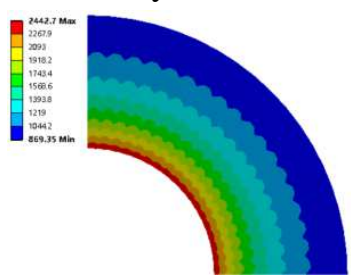

(u) Lattice 11

centrosymmetric

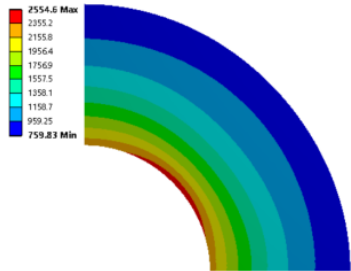

(r) Lattice 9 parallel

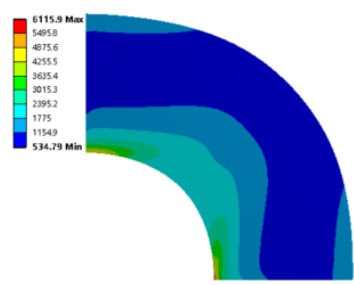

(v) Lattice 11 parallel

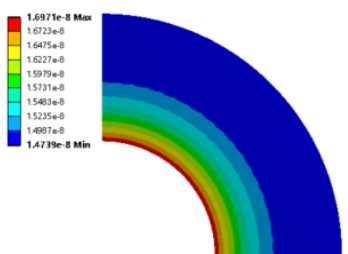

(a) Lattice 1 centrosymmetric

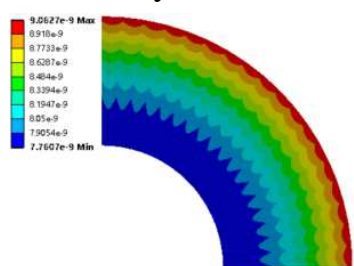

(e) Lattice 3

centrosymmetric

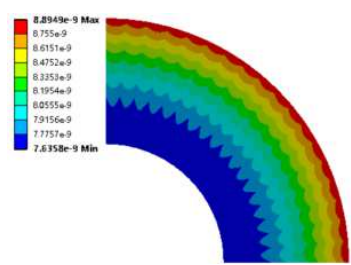

(i) Lattice 5

centrosymmetric

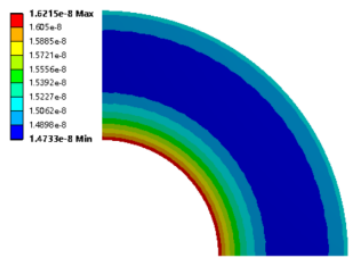

(m) Lattice 7 centrosymmetric

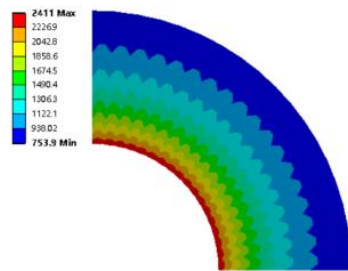

(s) Lattice 10 centrosymmetric

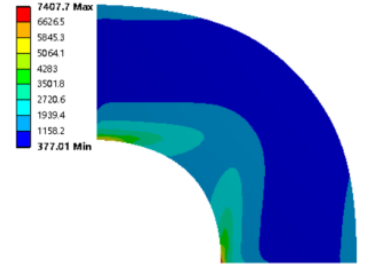

(t) Lattice 10 parallel

Figure 6 Stress distribution $(\mathrm{Pa})$

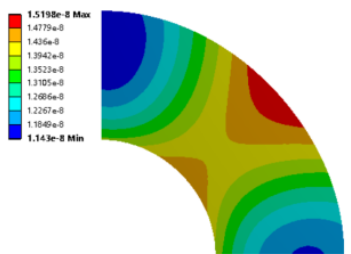

(b) Lattice 1 parallel

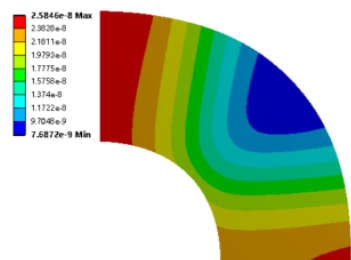

(f) Lattice 3 parallel

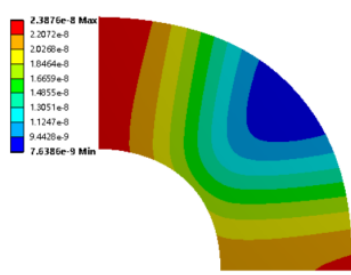

(j) Lattice 5 parallel

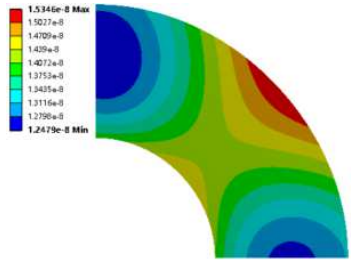

(n) Lattice 7 parallel

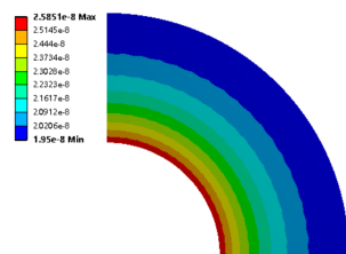

(c) Lattice 2 centrosymmetric

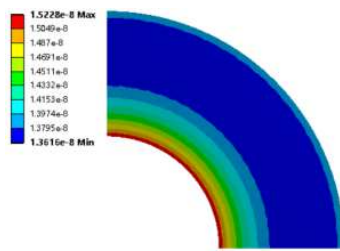

(g) Lattice 4 centrosymmetric

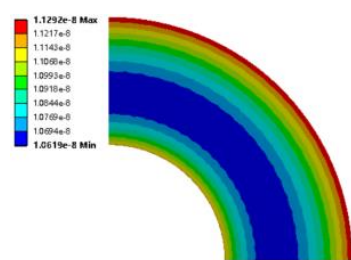

(k) Lattice 6 centrosymmetric

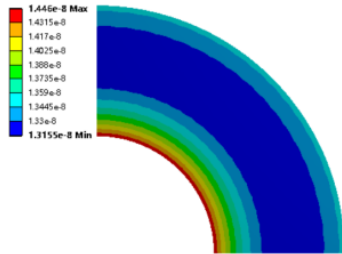

(o) Lattice 8 centrosymmetric

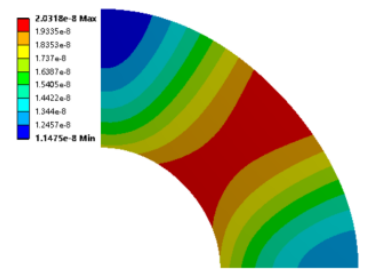

(d) Lattice 2 parallel

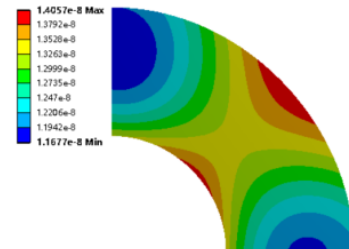

(h) Lattice 4 parallel

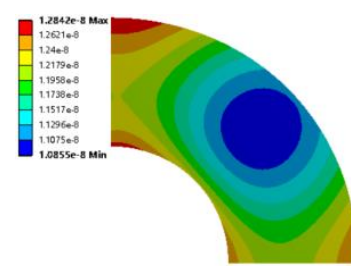

(1) Lattice 6 parallel

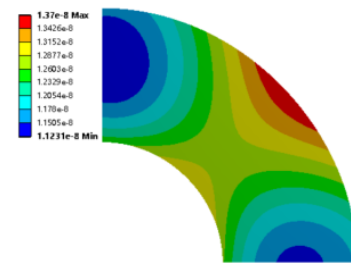

(p) Lattice 8 parallel 


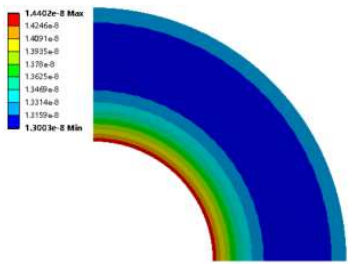

(q) Lattice 9

centrosymmetric

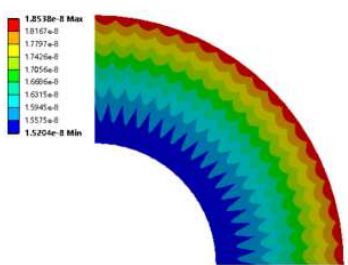

(u) Lattice 11

centrosymmetric

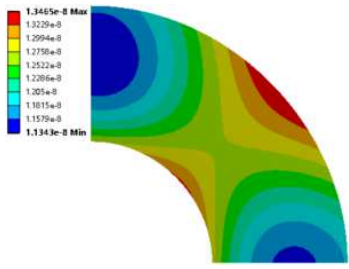

(r) Lattice 9 parallel

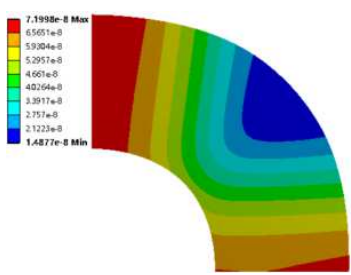

(v) Lattice 11 parallel

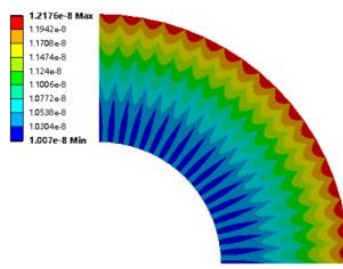

(s) Lattice 10 centrosymmetric

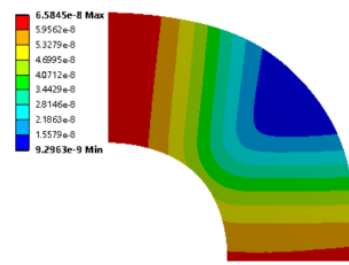

(t) Lattice 10 parallel

Figure 7 Displacement distribution (m)

3 Finite element analysis and structural performance evaluation of two concentric cylinders with centrosymmetric lattice

It can be seen from the second section that compared with the concentric cylinder with parallel array lattice, when the centrosymmetric lattice is applied to the structural lightweight design of concentric cylinder, the concentric cylinder can have a more uniform stress distribution and displacement distribution when only subjected to axisymmetric load. In Section 2, the homogenization method is used to analyze the static response of the lattice concentric cylinder. In this section, the centrosymmetric lattice concentric cylinder will be modeled directly instead of using the homogenization model to replace it. Then, the solid element with intermediate nodes is used to mesh the concentric cylinder, and the finite element method is used to analyze its static response under axisymmetric load. Here, we select two kinds of lattice commonly used in structural lightweight design, and apply them to the lightweight design of concentric cylinder. In the lightweight concentric cylinder, each column in the selected lattice is treated with variable section. Figure 8 (a) shows the concentric cylinder of centrosymmetric lattice with No.3 lattice for lightweight design, and Figure 8 (c) shows the concentric cylinder of centrosymmetric lattice with No.1 lattice for lightweight design. In order to impose boundary constraints on the finite element model, the quarter cylinder model will be selected in this section, and the models obtained are shown in Figure 8 (b) and Figure 8 (d) respectively. In addition, a path in Figure 9 (a) is selected to study the radial stress variation on the path under axisymmetric load. Figure 9 (b) shows the constraint and load of a quarter centrosymmetric lattice concentric cylinder under the action of axisymmetric plane pressure. 


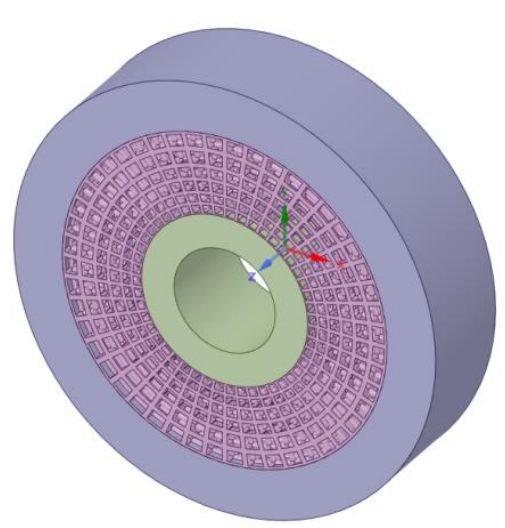

(a)

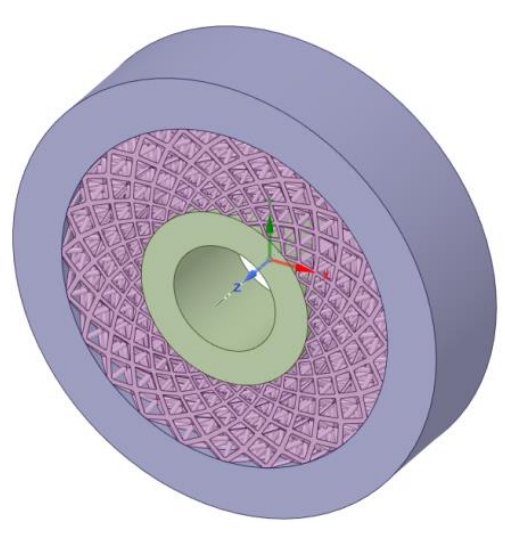

(c)

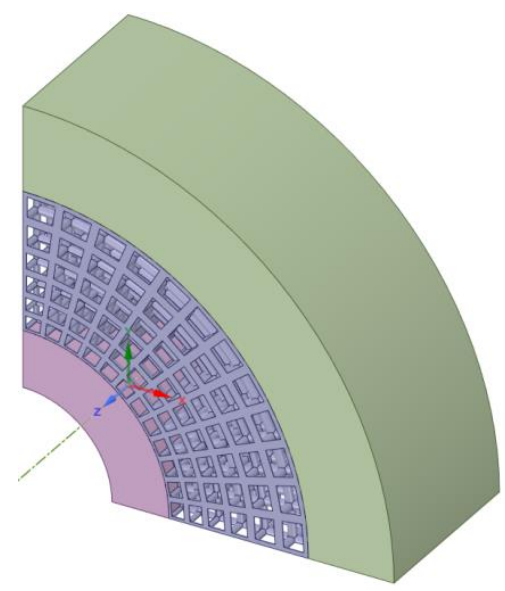

(b)

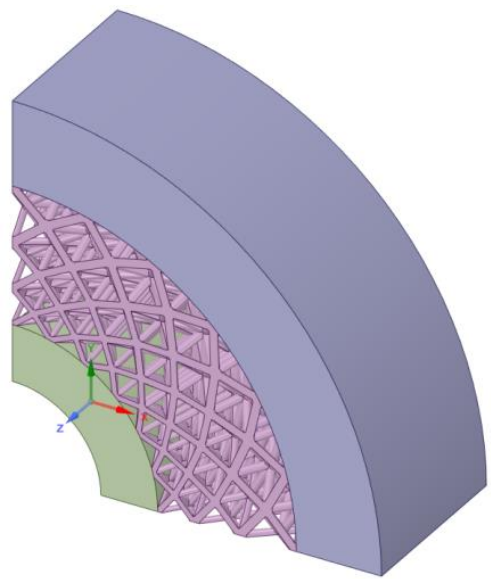

(d)

Figure 8 Lightweight lattice cylinder

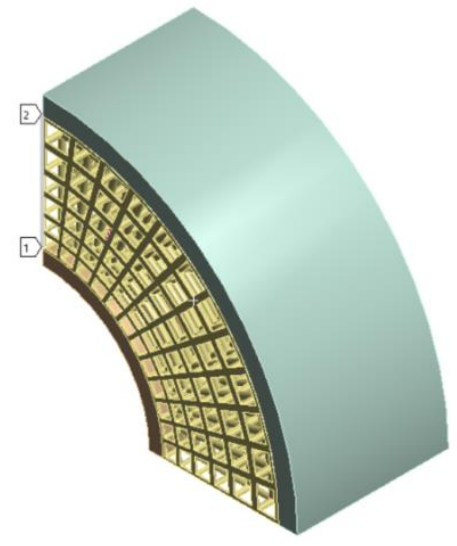

(a)

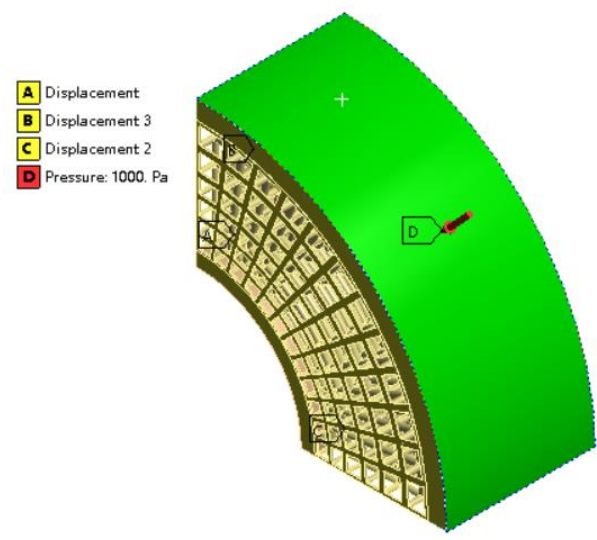

(b)

Figure 9 Specified stress path and loads

3.1 Static response analysis of centrosymmetric lattice concentric cylinder structure with No.3 lattice for lightweight design

Considering that the percentage of lattice structure in the concentric cylinder will affect the structural response of the concentric cylinder, this section parameterizes the thickness of the inner 
non lattice part (denoted as "inside") and the outer non lattice part (denoted as "outside") of the concentric cylinder. Figure 10 shows the maximum stress and displacement of the inside, the outside, and the the centrosymmetric lattice concentric cylinder with different inside thickness and different outside thickness under the axisymmetric pressure. In this case, the lattice form of concentric cylinder is No.3 lattice.

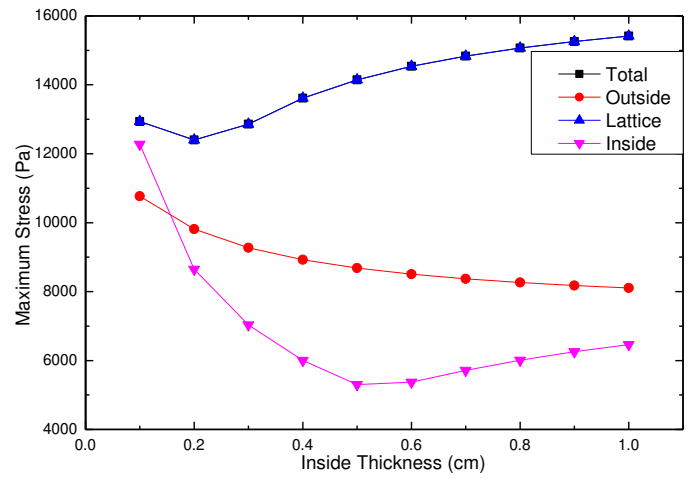

(a)

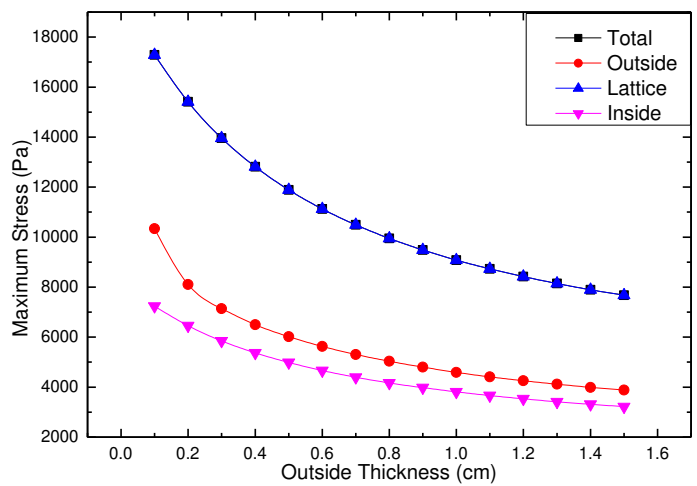

(c)

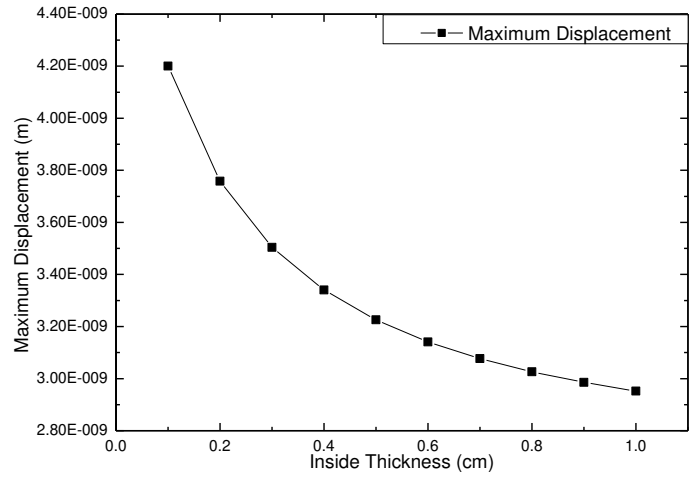

(b)

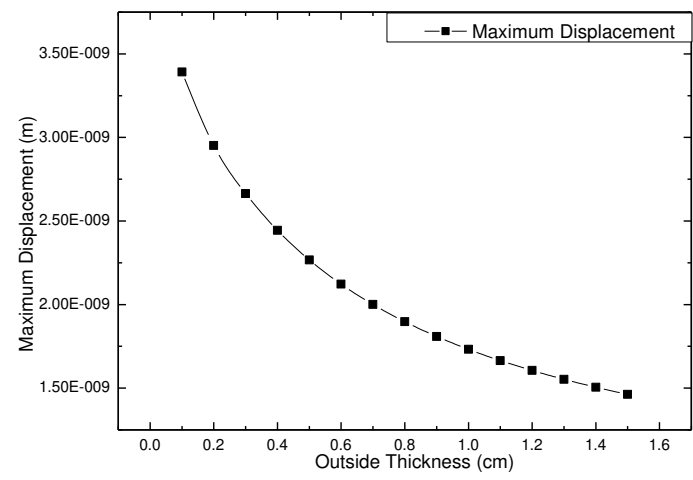

(d)

Figure 10 Maximum stress and displacement of a centrosymmetric lattice cylinder of No.3

It can be seen from Figure 10 (a) that the maximum structural stress of concentric cylinder with centrosymmetric lattice appears in the metal lattice region, and the maximum stress first decreases with the increase of inside thickness, and then increases with the increase of inside thickness. The maximum stress of the outside decreases with the increase of the inside thickness, and the maximum stress of the inside first decreases with the increase of the inside thickness, then, when the inside thickness exceeds $0.5 \mathrm{~cm}$, the maximum stress of the inside increases with the increase of the inside thickness. When the thickness of the outside increases, the maximum stress of the inside, the maximum stress of the inside and the maximum stress of the lattice region decrease with the increase of the thickness of the outside. It can be seen that for the concentric cylinder with centrosymmetric lattice, the influence of the outside thickness on the maximum stress is significant. In addition, it can be seen from Figure 10 (b) and Figure 10 (d) that the maximum displacement of concentric cylinder with centrosymmetric lattice will decrease with the increase of outside thickness or inside thickness, which is also consistent with the objective reality.

In addition, in order to study the force transfer effect of the lattice structure between the outside and the inside of concentric cylinder, this section calculates the difference between the maximum stress of the outside and the maximum stress of the inside, the difference between the maximum stress of the outside and the maximum stress of the lattice structure, and the difference 
between the maximum stress of the inside and the maximum stress of the lattice structure (Figure 11 (b)). Besides, this section also calculates the difference between the maximum stress values of the innermost and outermost concentric cylinder homogenization models corresponding to the 11 lattice listed in Section 2 under axisymmetric pressure (Figure 11 (c)).

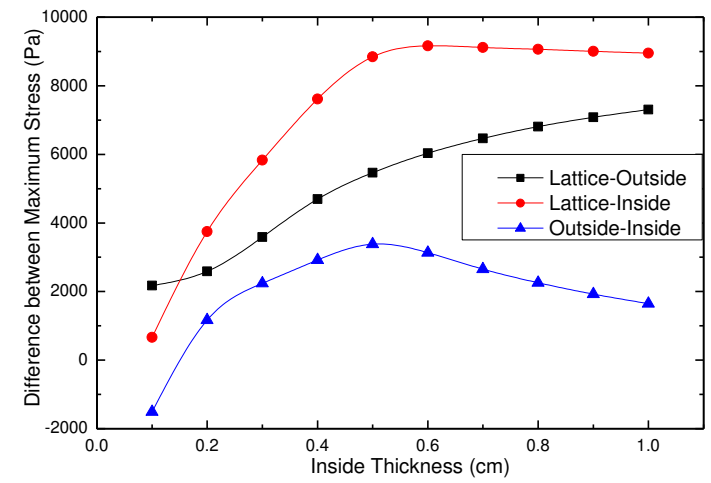

(a)

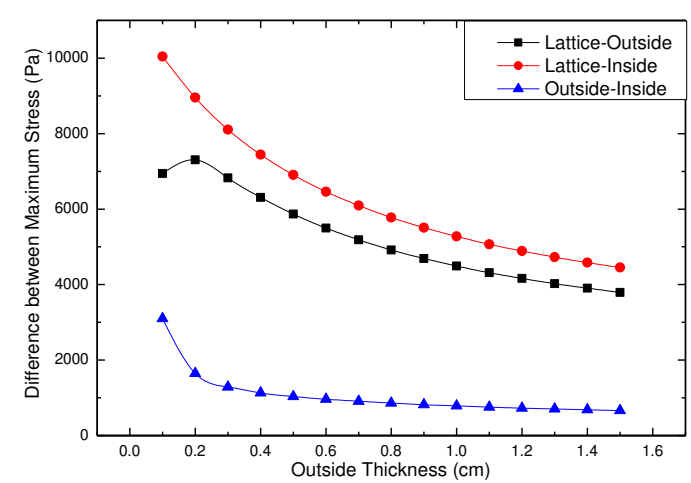

(b)

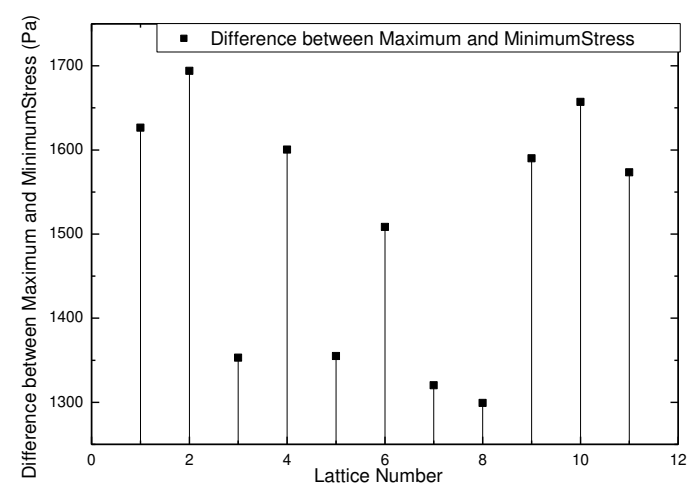

(c)

Figure 11 Stress difference

It can be seen from Figure 11 (a) and Figure 11 (b) that with the increase of the thickness of the inside of the concentric cylinder with centrosymmetric lattice structure, the difference between the maximum stress of the lattice structure and the maximum stress of the inside, the difference between the maximum stress of the lattice structure and the maximum stress of the outside will gradually increase, and the maximum stress of the outside is obviously higher than the maximum stress of the inside. It can be seen that increasing the inside thickness can reduce the force transfer effect of lattice structure. When the thickness of the inside is $0.1 \mathrm{~cm}$, the maximum stress of the outside is lower than that of the inside; when the inside thickness is between $0.2-0.5 \mathrm{~cm}$, the difference between the maximum stress of the outside and the maximum stress of the inside will increase with the increase of the inside thickness; then, when the inside thickness exceeds $0.6 \mathrm{~cm}$, the difference between the maximum stress of the outside and the maximum stress of the inside will decrease with the increase of the inside thickness.

It can be seen from Figure 11 (c) and Figure 6 that for the homogenized 11 kinds of lattice concentric cylinders, the maximum and minimum structural stress are usually distributed in the outside and the inside of the cylinder, respectively, and the difference between the maximum and the minimum is between $1300 \mathrm{~Pa}$ and $1700 \mathrm{~Pa}$. This result is in good agreement with the static response of the non-uniform concentric cylinder with centrosymmetric lattice by solid modeling 
and finite element analysis. In order to observe the stress distribution of concentric cylinder with centrosymmetric lattice more intuitively, this section outputs the stress distribution diagrams of four kinds of concentric cylinder with centrosymmetric lattice, which have different inside thickness and outside thickness are lightweight designed by using lattice No. 3. In this section, four types of centrosymmetric lattice concentric cylinders with thicker inside and outside (Figure 12 (a)), thicker inside and thinner outside (Figure 12 (b)), thinner inside and thicker outside (Figure 12 (c)) and thinner inside and outside (Figure 12 (d)) are selected. According to the stress distribution of each concentric cylinder, when the thickness of the inside increases, the external surface pressure transferred to the inside of the concentric cylinder is limited. Therefore, the larger the thickness of the inside of the concentric cylinder, the larger the area of low stress in the inside. In addition, the thickness of the outside of the concentric cylinder will have a significant impact on the stress distribution of the concentric cylinder: the greater the thickness of the outside, the greater the low stress area of the outside. However, because the outside bears the external load directly, the minimum stress in the low stress region of the outside is obviously higher than that in the low stress region of the inside. For the concentric cylinder with No.3 lattice, the stress of the radial column is obviously higher than the maximum stress of the circumferential column. This result is consistent with the mechanical response of parallel lattice structure under tension compression load.

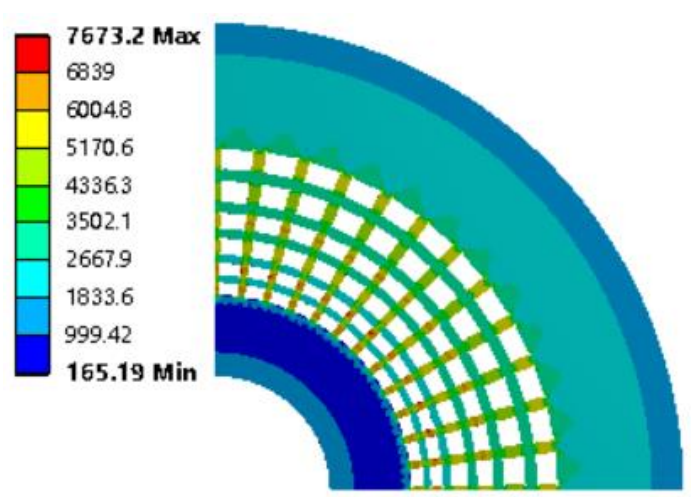

(a)

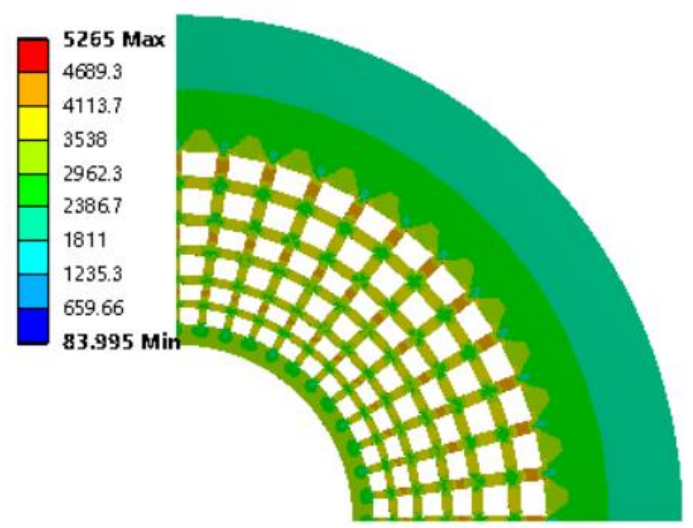

(c)

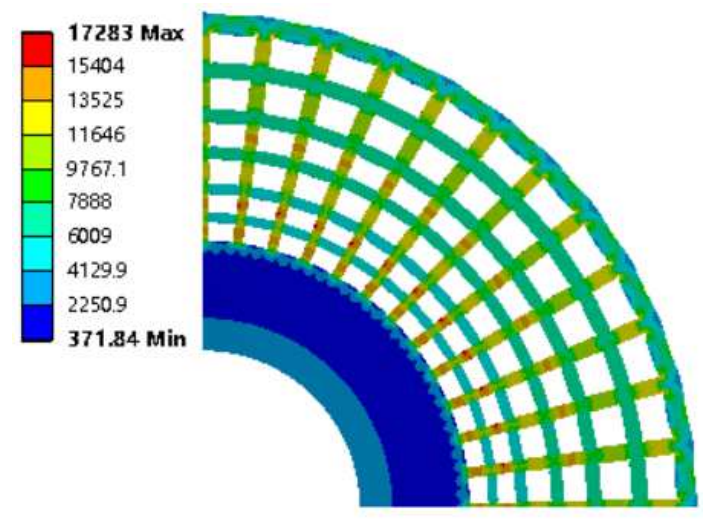

(b)
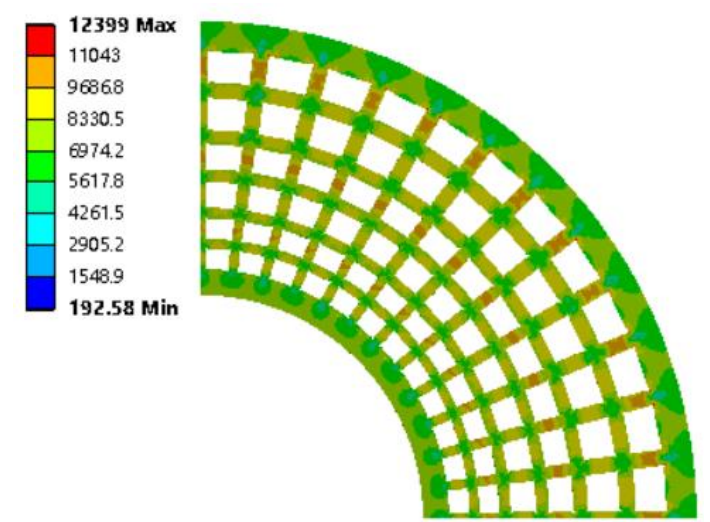

(d)

Figure 12 Stress distribution $(\mathrm{Pa})$

3.2 Static response analysis of centrosymmetric lattice concentric cylinder structure with No.1 lattice for lightweight design 
Similar to Section 3.1, this section studies the static response of concentric cylinder with No.1 lattice under axisymmetric pressure.

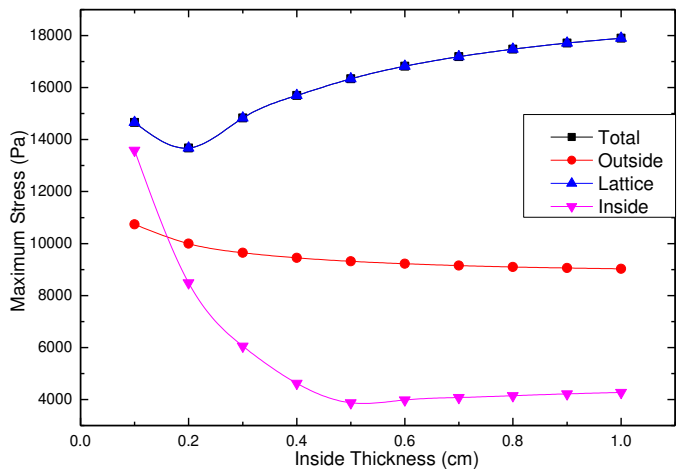

(a)

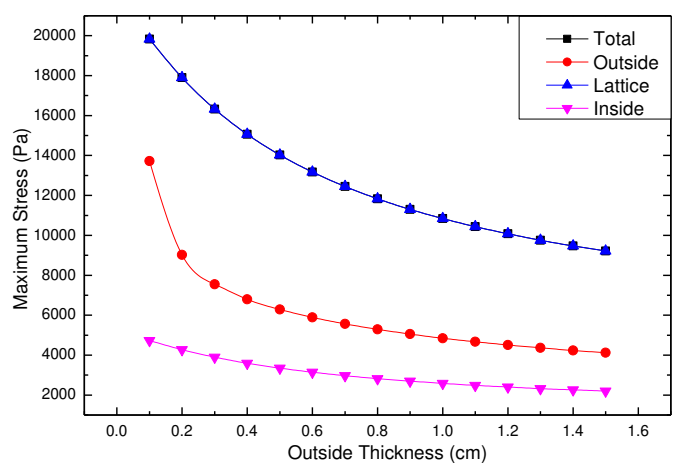

(c)

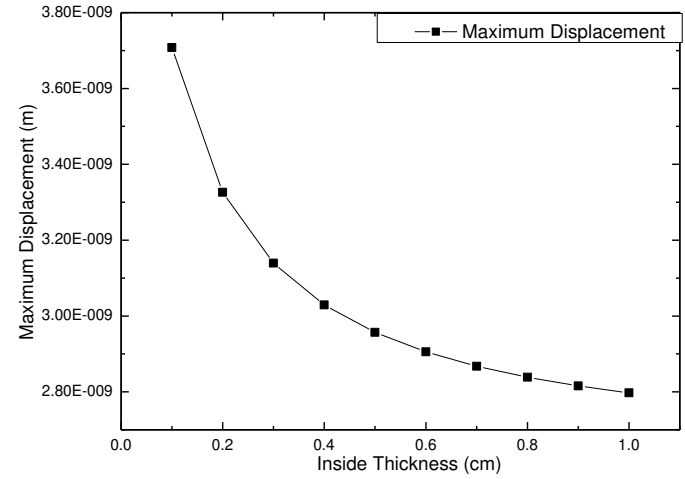

(b)

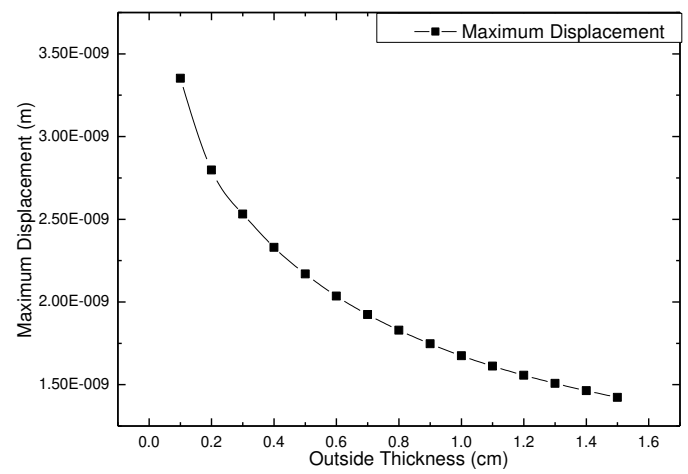

(d)

Figure 13 Maximum stress and displacement of a centrosymmetric lattice cylinder of No.1

Figure 13 shows the maximum stress and displacement of the inside, the maximum stress and displacement of the outside, and the maximum stress and displacement of the lattice region of a concentric cylinder with different inside thickness and different outside thickness under axisymmetric load. In this case, No.1 lattice is used to lightweight the concentric cylinder. It can be seen from Figure 13 (a) that when the No. 1 lattice is applied to the lightweight design of the concentric cylinder, the maximum stress of the structure appears on the metal lattice, and the maximum stress first decreases with the increase of the inside thickness, and then increases with the increase of the inside thickness. The maximum stress of the outside decreases with the increase of the inside thickness. The maximum stress of the inside first decreases with the increase of the inside thickness, and then increases with the increase of the inside thickness when the inside thickness exceeds $0.5 \mathrm{~cm}$. When the thickness of the outside increases, the maximum stress of the outside, the maximum stress of the inside and the maximum stress of the lattice region decrease with the increase of the thickness of the outside. It can be seen that the thickness of the outside has a significant effect on the stress of the whole concentric cylinder when the No.1 lattice is applied to the lightweight design of the concentric cylinder. In addition, it can be seen from Figures 13 (b) and 13 (d) that the maximum displacement of the centrosymmetric lattice cylinder will decrease with the increase of the outside thickness or the inside thickness. This conclusion is also consistent with the objective reality. In addition, in order to study the force transfer effect from the outside to the inside of concentric cylinder with centrosymmetric lattice in lightweight design, this section calculates the difference between the maximum stress of the outside and the maximum stress of 
the inside of concentric cylinder with different outside thickness and inside thickness, the difference between the maximum stress of the outside and the maximum stress of the lattice area, and the change of the difference between the maximum stress of the inside and the maximum stress of the lattice area with the change of the thickness of the inside and the change of the thickness of the outside (Figure 14). It can be seen from Figure 14 that, in terms of stress distribution, deformation distribution and force transfer effect, the change of lattice form does not affect the qualitative change trend of the above results, but has a significant impact on the maximum value of stress distribution.

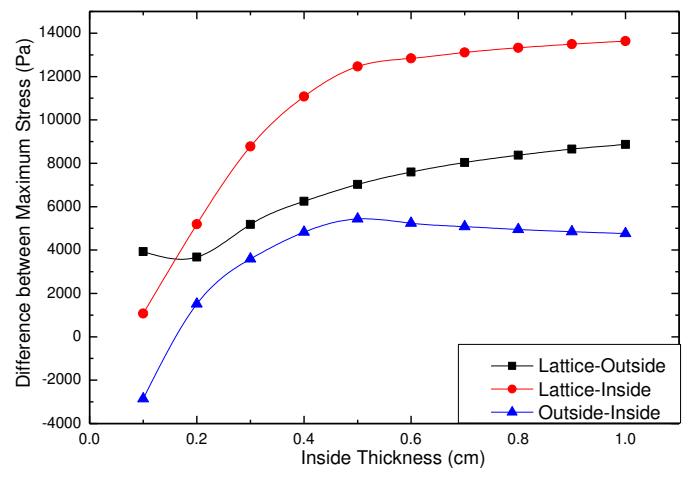

(a)

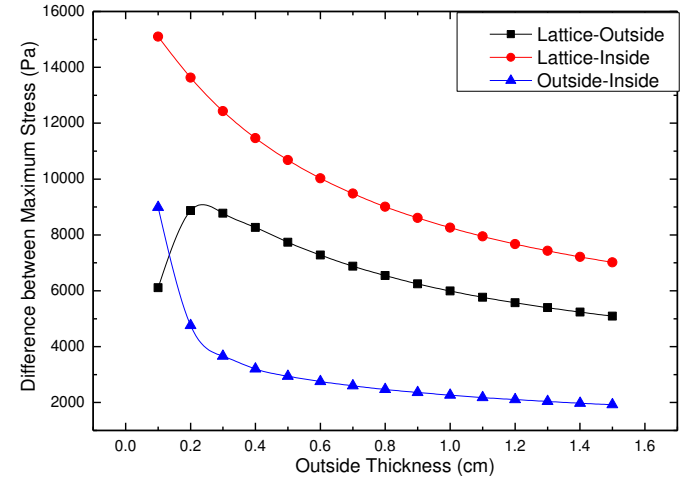

(b)

Figure 14 Stress difference

According to Figure 14 and Figure 6, for the mechanical response of the concentric cylinder homogenization model with 11 kinds of lattice for lightweight design under axisymmetric pressure, the maximum and minimum structural stress are usually distributed in the outside and the inside of the concentric cylinder, and the difference between the maximum and minimum values is between $1300 \mathrm{~Pa}$ and $1700 \mathrm{~Pa}$. This is in good agreement with the stress calculation results of concentric cylinder with centrosymmetric lattice. In order to study the stress distribution of the inhomogeneous model of solid centrosymmetric lattice concentric cylinder under axisymmetric pressure, this section outputs the stress distribution diagrams of four centrosymmetric lattice concentric cylinders with different inside and outside thicknesses. The four cylinders are designed with No.1 lattice. The inside thickness and outside thickness of the four cylinders are: thicker inside and outside (Figure 15 (a)), thicker inside and thinner outside (Figure 15 (b)), thinner inside and thicker outside (Figure 15 (c)), and thinner inside and outside (Figure 15 (d)). From the stress distribution diagram of each cylinder, it can be seen that for the concentric cylinder with No.1 lattice for lightweight design, the stress transmitted by the inside of the cylinder is very limited. Therefore, the larger the thickness of the inside of the concentric cylinder, the larger the area of low stress in the inside. The thickness of the outside of the concentric cylinder will also affect the structural stress distribution of the cylinder: the thicker the outside is, the larger the low stress area of the outside will be. However, because the outside bears the external load directly, the minimum stress in the low stress region is obviously higher than that in the inside. For the concentric cylinder with No.1 lattice for lightweight design, the stress of the column is significantly higher than that of the junction point of the column. 


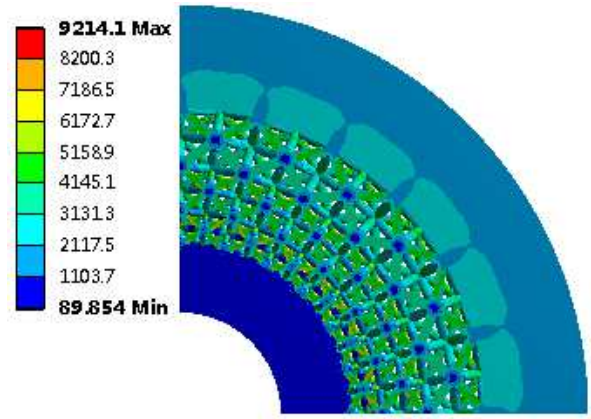

(a)

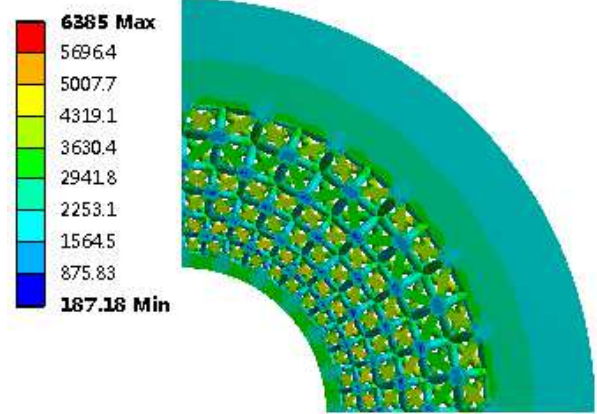

(c)
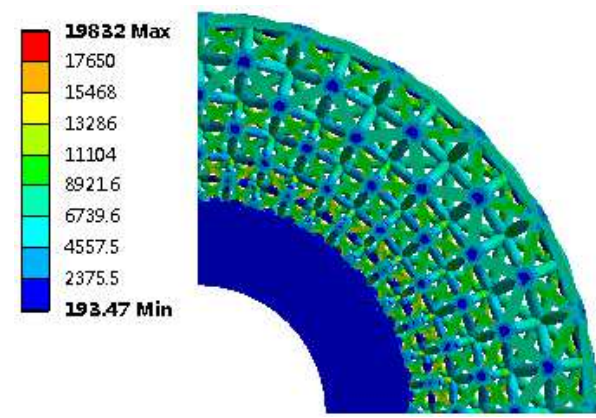

(b)
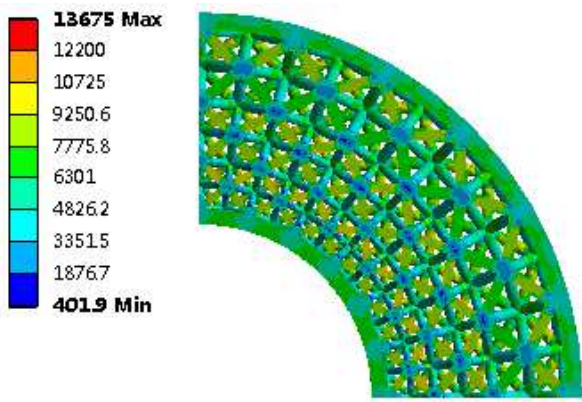

(d)

Figure 15 Stress distribution $(\mathrm{Pa})$

3.3 Influence of lattice column size on mechanical response of concentric cylinder with centrosymmetric lattice

The influence of the thickness of outside and inside on the static response of concentric cylinder with centrosymmetric lattice under axisymmetric load is described in the previous paper, i.e. the influence of the size of lightweight region on the static response of concentric cylinder is analyzed and discussed. In this section, we will discuss the influence of the column size of lattice on the static response of concentric cylinder with centrosymmetric lattice. Figure 16 shows the maximum stress, path stress and displacement of concentric cylinder with different lattice cross-section sizes under axisymmetric pressure. Here, the axisymmetric load is the load initially applied to the outer surface of the concentric cylinder in this section. The path is shown in Figure 9 (a), and the lightweight lattice is No. 3 lattice.

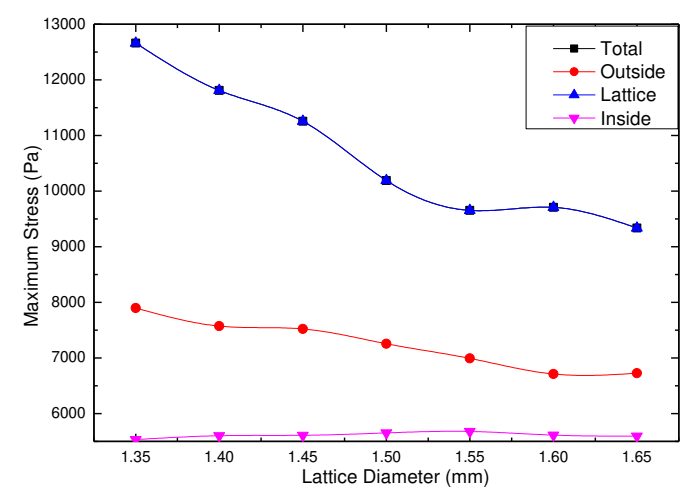

(a)

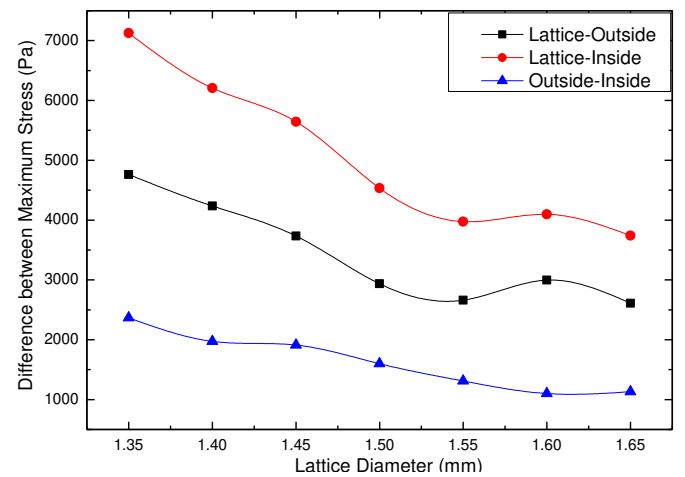

(b) 


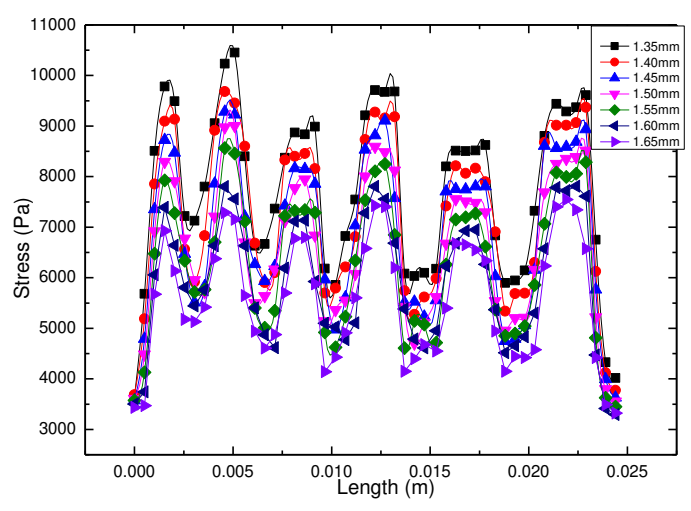

(c)

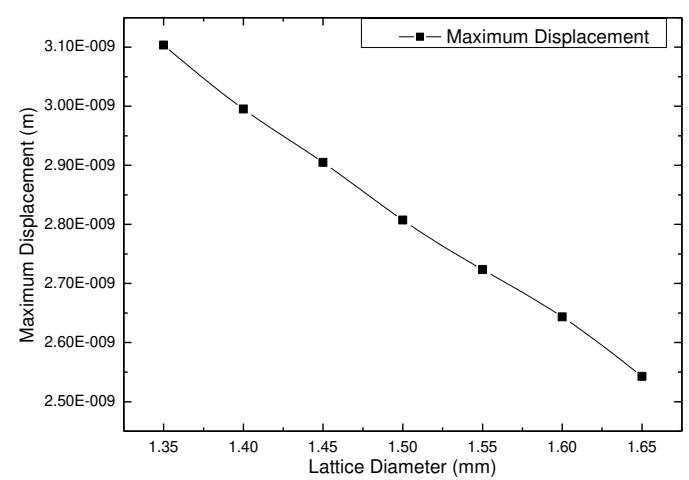

(d)

Figure 16 The maximum displacement, maximum stress and path stress of lattice concentric cylinders with different cross-section sizes

It can be seen from Figure 16 (a) that with the increase of the cross-section diameter of the lattice column, the maximum value of the inside of the concentric cylinder will change in a small range, while the maximum value of the outside stress and the lattice stress will change obviously. With the increase of section diameter of lattice column, the outside stress and lattice stress decrease obviously, and the maximum value of stress decreases fastest when the lattice stress is between 1.35-1.55 $\mathrm{mm}$ in diameter. Figure 16 (b) shows the difference between the maximum stress of the lattice structure and the maximum stress of the outside, the difference between the maximum stress of the lattice structure and the maximum stress of the inside, and the difference between the maximum stress of the outside and the maximum stress of the inside. With the increase of the cross-section diameter of the lattice, the difference also decreases. It can be seen from Figure 16 (c) that the maximum stress of concentric cylinder with centrosymmetric lattice appears at the non node, and the stress value at the non node is significantly higher than that at the node. When using No.1 lattice to lightweight design the concentric cylinder with central symmetric lattice, we can consider improving the structure, increasing the possibility of node bearing and reducing the possibility of column bearing, so as to make the stress distribution of the whole concentric cylinder more uniform and reduce the phenomenon of stress concentration. It can be seen from Figure 16 (d) that when the lattice cross-section diameter of the concentric cylinder with centrosymmetric lattice increases, its structural displacement will decrease. This conclusion is also consistent with the objective facts. Different from the trend of stress change, the displacement of concentric cylinder decreases linearly. When the diameter of lattice section increases, the equivalent structural stiffness of concentric cylinder lattice increases. In this case, the stress and displacement of the concentric cylinder will increase. In other words, in a certain range of parameters, when the lattice is applied to the lightweight design of the structure, the structure will produce adverse static response. In the lightweight design of axisymmetric structure, how to select the lattice parameter range will be the focus of future research.

\section{Discussion and Conclusion}

In this paper, 11 kinds of metal lattice are symmetrized, and the centrosymmetric lattice is applied to the lightweight design of concentric cylinder. Based on the finite element method, the finite element analysis of concentric cylinder with centrosymmetric lattice under axisymmetric load is carried out, and the influence of geometric parameters on the mechanical response of 
concentric cylinder is evaluated. The results show that:

(1) The results of finite element analysis based on homogenization method show that the stress of concentric cylinder with centrosymmetric lattice is uniformly distributed along the circumference when subjected to axisymmetric load. In most cases, the displacement of the structure is uniformly distributed along the circumference; in some cases, the distribution along the circumference is periodic. For the concentric cylinder with parallel array lattice, the stress distribution is not uniform along the circumferential and radial direction when it is subjected to axisymmetric load, the distribution of structural displacement is also highly similar.

(2) When No.3 lattice (pure column lattice) is applied to the lightweight design of concentric cylinder with centrosymmetric lattice, the stress of each part of the concentric cylinder decreases with the increase of the outside thickness, and the larger the outside is, the less obvious the change of the maximum stress is. When the percentage of lattice area increases, the maximum stress of concentric cylinder decreases sharply at first, then increases slightly, and fluctuates in a stable range. The stress distribution of concentric cylinder with No.1 lattice (octagonal cubic lattice) for lightweight design is similar.

(3) With the increase of the inside thickness, the difference of the maximum stress between the outside and the inside, the difference of the maximum stress between the outside and the lattice region, and the difference of the maximum stress between the lattice region and the inside increase with the increase of the inside thickness. When the inside thickness is greater than $0.5 \mathrm{~cm}$, the difference between the maximum stress of the outside and the inside will decrease with the increase of the inside thickness. This difference will change in a relatively stable range.

(4) For the concentric cylinder with centrosymmetric lattice, the stress at the intersection of columns is less than that at the non intersection, and the difference is very obvious. Therefore, in the subsequent lightweight design of centrosymmetric lattice axisymmetric structure, we can consider reducing the difference by improving the structure form and geometric size of the lattice.

(5) For the centrosymmetric lattice concentric cylinders with different cross-section sizes of lattice columns, which are designed with pure column lattice for lightweight design, when the cross-section diameter increases, the maximum external stress of the concentric cylinder and the maximum stress in the lattice region will decrease, but the maximum internal stress of the cylinder does not change much. The increase of the cross-section diameter of the lattice will promote the force transfer on the cylinder.

In this paper, the mechanical response of concentric cylinder with centrosymmetric lattice is discussed. The conclusions and methods of this paper can provide reference for the future research on parametric design, optimization and parameter sensitivity analysis of axisymmetric structures.

\section{References:}

[1] Wu L, Xiong J, Ma L, et al. Processes in the study on novel composite sandwich panels with lattice truss cores [J]. Advances in Mechanics, 2012, 42(1): 41-66.

[2] $\mathrm{Wu} \mathrm{L}$, Xiong J, Ma L, et al. Intergrated design of lightweight multifunctional sandwich structures [J]. Mechanics in Engineering, 2012, 34(4): 8-18.

[3] Guo Z, Liu C, Li F. Vibration analysis of sandwich plates with lattice truss core [J]. Mechanics of Advanced Materials and Structures, 2017: 1-6.

[4] Seepersad C C, Dempsey B, Allen J K, et al. Design of multifunctional honeycomb materials [J]. AIAA Journal, 2004, 42(5): 1025-1033. 
[5] Lee M G, Hoang V M, Yoon J W, et al. Compressive strength of wire-woven bulk kagome with various orientations [J]. Procedia Materials Science, 2014, 4: 209-214.

[6] Barnett D, Rawal S, Kevin R. Multifunctional structures for advanced spacecraft [J]. Journal of Spacecraft \& Rockets, 2015, 38(2): 226-230.

[7] Huang W, Zhang W, Li D, et al. Dynamic failure of honeycomb-core sandwich structures subjected to underwater impulsive loads [J]. European Journal of Mechanics - A / Solids, 2016, 60: 39-51.

[8] Kim T, Hodson H P, Lu T J. Fluid-flow and end wall heat-transfer characteristics of an ultralight lattice-frame material [J]. International Journal of Heat and Mass Transfer, 2004, 47(6-7): 1129-1140.

[9] Xie G, Wang Q, Sunden B, et al. Thermomechanical optimization of lightweight thermal protection system under aerodynamic heating [J]. Applied Thermal Engineering, 2013, 59(1-2): 425-434.

[10] Liu Y, Daudin C. Analytical modelling of sound transmission through finite clamped double-wall sandwich panels lined with poroelastic materials [J]. Composite Structures, 2017, 172: 359-373.

[11] Young V, Querin O M, Steven G P. 3D and multiple load case bi-directional evolutionary structrural optimization (BESO) [J]. Structural Optimization, 2006, 18.

[12] Gibson R F. A review of recent research on mechanics of multifunctional composite materials and structures [J]. Composite Structures, 2010, 92(12): 2793-2810.

[13] Yin L, Doyhamboure--Fouquet J, Tian X, et al. Design and characterization of radar absorbing structure based on gradient-refractive-index metamaterials [J]. Composites Part B: Engineering, 2018, 132: 178-187.

[14] Evans A G, Hutchinson J W, Fleck N A, et al. The topological design of multifunctional cellular metals [J]. Progress in Materials Science, 2001, 46(3-4): 309-327.

[15] Jia D, Li F, Zhang C, et al. Design and simulation analysis of Lattice bone plate based on finite element method [J]. Mechanics of Advanced Material and Structures, 2020, DOI: 10.1080/15376494.2019.1665759

[16] Zhang Y, Li F, Jia D. Lightweight design and static analysis of lattice compressor impeller [J]. Scientific Reports, 2020, 10: 18394.

[17] Zhang Y, Li F, Jia D. Residual stress and deformation analysis of lattice compressor impeller based on 3D printing simulation [J]. Mechanics of Advanced Material and Structures, 2020, DOI: 10.1080/15376494.2020.1789926.

[18] Zhang Y, Li F, Jia D. Lattice impeller design and multi-scale stress-deformation analysis based on conventional cubic lattice [J]. Mechanics of Advanced Material and Structures, 2020, DOI: $10.1080 / 15376494.2020 .1855681$.

[19] Wadley H N. Multifunctional periodic cellular metals [J]. Philos. Trans. R. Soc. A, 2020, 364: $31-68$.

[20] Ashby, M. Hybrid materials to expand the boundaries of material-property space [J]. J. Am. Ceram. Soc. 2011, 94: s3-s14.

[21] Gibson L J, Ashby M F, Harley B A. Cellular Materials in Nature and Medicine (Cambridge Univ. Press, 2010).

[22] Tao W, Leu M C. Design of lattice structure for additive manufacturing [Z]. IEEE, 2016325-332. 
[23] Engelbrecht S, Folgar L, Rosen D W, et al. Cellular structures for optimal performance [Z]. 2009831-842.

[24] Corney J. 3D Modeling using the ACIS Kernel and Toolkit [M]. John Wiley \& Sons, Inc., 1997.

[25] Wang H, Chen Y, Rosen D W. A hybrid geometric modeling method for large scale conformal cellular structures [Z]. 200524-28.

[26] Berger J, Wadley H N G, Mcmeeking R M. Mechanical metamaterials at the theoretical limit of isotropic elastic stiffness [J]. Nature, 2017, 543(7646): 533-+.

[27] Wang L, Kang J, Sun C, et al. Mapping porous microstructures to yield desired mechanical properties for application in 3D printed bone scaffolds and orthopaedic implants [J]. Materials \& Design, 2017, 133: 62-68.

[28] Yang Y, Shana M, Zhao L, et al. Multiple strut-deformation patterns based analytical elastic modulus of sandwich BCC lattices [J]. Materials \& Design, 2019, 181: 107916. 